\title{
Method and Apparatus for a Differential Localized Microscopy System Based on Position Sensitive Detector

\author{
Author(s): ${ }^{1}$ Venky Dijkkomp, ${ }^{* 1}$ Kanai Ogale \\ National University of Singapore \\ *Corresponding Author: KanaiOgale@outlook.com
} \\ Affiliation(s): ${ }^{1}$ Department of Integrative Sciences and Engineering
}

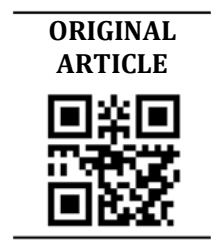

\begin{abstract}
A precise measurement of position using a Position Sensitive Detector (PSD) is fundamental in mitigating the geometric error factors that are caused by the pincushion-type distortion of these sensors. These errors can be addressed by implementing a differential localized method to significantly reduce signal to noise ratio (SNR) in PSD and the microscopy system. The differential method based on Time difference of Arrival (TDoA) is proposed and implemented in this research. The simulation and the actual results of the system further confirm the significant improvement in accuracy and precision of the system.
\end{abstract}

Keywords: Position Sensitive Detector (PSD); Microscopy systems; Sensor calibration; Localized differential method

\section{INTRODUCTION}

The Position Sensitive Detector (PSD) is an optical position sensor (OPS) capable of measuring the position of a light spot in one or two-dimensions on the sensor surface. The PSD has been employed in many applications but the most famous one is in the Atomic Force Microscopy (AFM) systems. The very high resolution of the PSD makes it suitable for such applications.

The challenges of conducting a precise measurement using the position sensitive detector (PSD) has been in investigation by researchers $[1,3,5]$. The most substantial factor in the calibration of a local positioning systems (LPS) is the differential phase shift between the receivers. One solution to this particular problem is in employing a phaselocked loop (PLL) suggested by Monzú et al [1]. Their approach is based on a measurement system that aimed at mitigating the noise effects using pseudorandom codes modulated by a sinusoidal signal to correct for the errors in position calculations. This is possible because of using a delay-locked loop (DLL).

Another approach was proposed by implementing a system for differential phase measurement [2]. These research are based on using the PSD in an open environment that transmit signal to an agent robot.

A more precise application was also investigated by researchers to construct a PSD microscopy system [3]. A significant improvement was reported to as implementing an accelerated adaptive local scanning but further results in this area are not suggesting a precise measurement.

The most novel approach in this regard is the development of a LPS sensor system where PSD sensors are used as IR signal detectors [5]. While another approach used a 2D bisection method by employing a DLP projector to scan the PSD area efficiently [6].

While these approaches have introduced significant advances to the measurement accuracy of the PSD, but still the SNR problem that causes the most important and boldest effect on the precision measurement of the PSD is left to be solved. The proposed method in this research is investigating a localized differential method to mitigate these challenges. The geometric calibration techniques that are used have significantly improved the overall accuracy and precision. In addition to the simulation performed in MATALB and PSpice, a model of the system was built to test the applicability of the proposed method. The presented results further confirmed the improvements that were seen in the computer simulation section.

\section{METHOD AND APPARATUS}

The PSD sensor principle of work is based on photodiode structured on either side of the PSD that generate a current 
dependent on the optical power of the light beam striking its active area. Fig. 1 shows the PSD sensor and an equivalent pin-cushion PSD circuit.

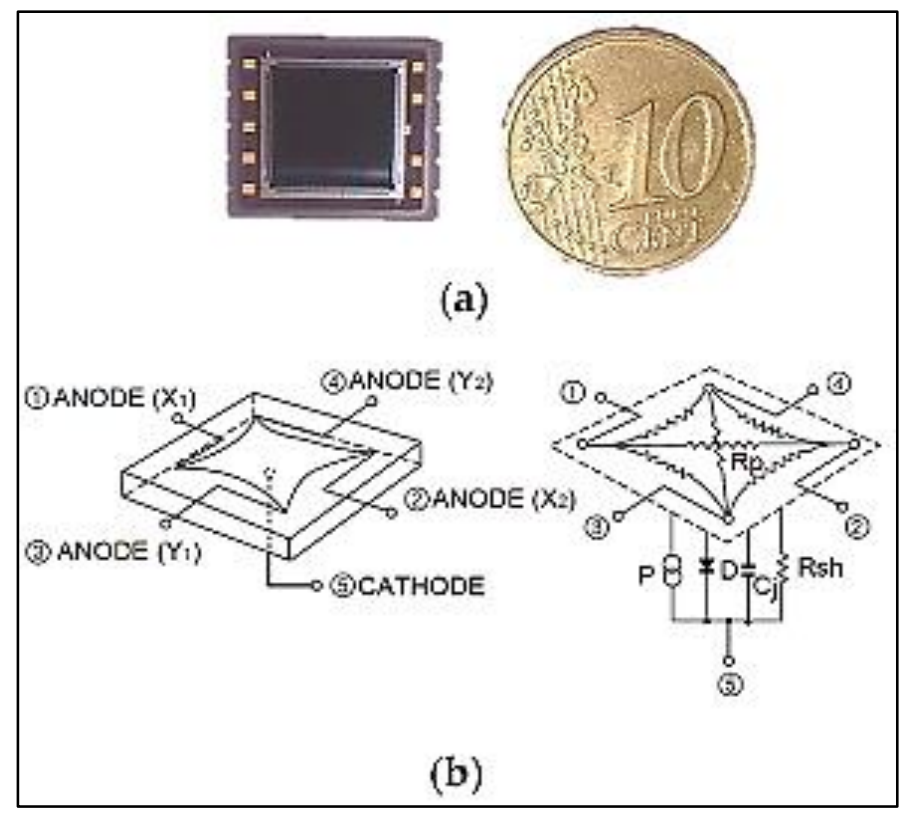

Figure 1. (a) Image of PSD S5991-01, Hamamatsu. (b) Equivalent pin-cushion PSD circuit (image courtesy of Hamamatsu, obtained from PSD technical information)

To better illustrate the function of the PSD, a sectional cut is presented. Fig. 2 shows a sectional cut of a one-dimensional PSD that uses a uniform P-type resistive layer. The 2-D tetra-lateral PSD works based on the same principle too.

The photocurrent that is received on either electrode can be calculated by the Eq. 1 .

$$
x=k_{x} \cdot \frac{I_{b}-I_{d}}{I_{b}+I_{d}}
$$

and

$$
y=k_{y} \cdot \frac{I_{a}-I_{c}}{I_{a}+I_{c}}
$$

where $I_{a-c}$ are the currents that are received on each of the electrodes on the sides of the PSD.

The non-linearity of the PSD was further improved by Song et al [7] as shown in Eq. 2. This has been employed to better estimate the incident light position. Although an improved approach was used in [8] be correction the position error using the Brown's distortion model [9].

This has mitigated the pincushion-type distortion as to strengthen the scanning lines of the PSD microscopy system proposed.

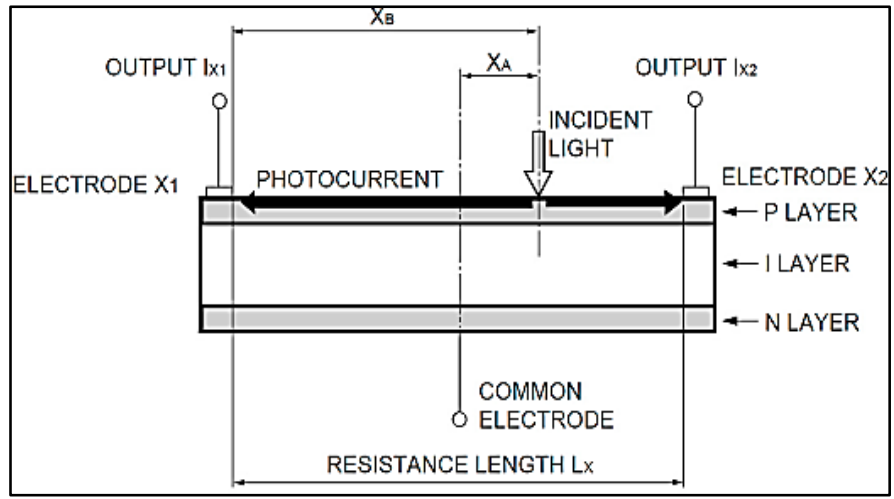

Figure 2. A sectional cut of a one-dimensional PSD is shown here. The incident light determines the photocurrent that the electrodes receive.

$$
\begin{aligned}
& x=k_{x 1} \cdot \frac{I_{4}-I_{3}}{I_{0}-1.02\left(I_{2}-I_{1}\right)} \cdot \frac{0.7\left(I_{2}+I_{1}\right)+I_{0}}{I_{0}+1.02\left(I_{2}-I_{1}\right)} \\
& y=k_{y 1} \cdot \frac{I_{2}-I_{1}}{I_{0}-1.02\left(I_{4}-I_{3}\right)} \cdot \frac{0.7\left(I_{4}+I_{3}\right)+I_{0}}{I_{0}+1.02\left(I_{4}-I_{3}\right)}
\end{aligned}
$$

This model is investigated by Villiers [10] and shown to rectify the pincushion-type distortion in other researches [11] in this area.

The transimpedance amplifier used in this research is shown in Fig. 3. The $I_{1}$ current source represents the PSD electrode photocurrent for one of the channels. The junction capacitance and the feedback resistance for the operational amplifier are ideal for the noise reduction purpose.

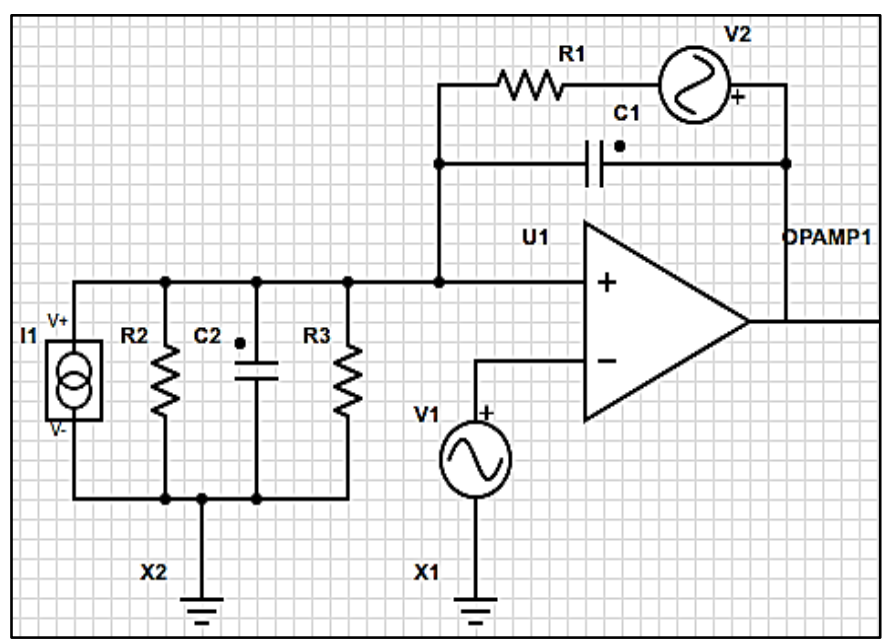

Figure 3. Equivalent PSD sensor circuit and transimpedance amplification circuit with noise is shown here. 
The thermal noise associated with the Johnson noise current is generated by inter-electrode resistance reported by the Hamamatsu PSD. These conditions represented a mostly conservative situation which would be unlikely to occur in a real case.

\section{SIMULATION RESULTS}

The simulation of the proposed system was implemented in MATLAB and PSpice to verify the model system. Fig. 4. shows the overall noise of the PSD system before applying the filtering systems designed in this study.

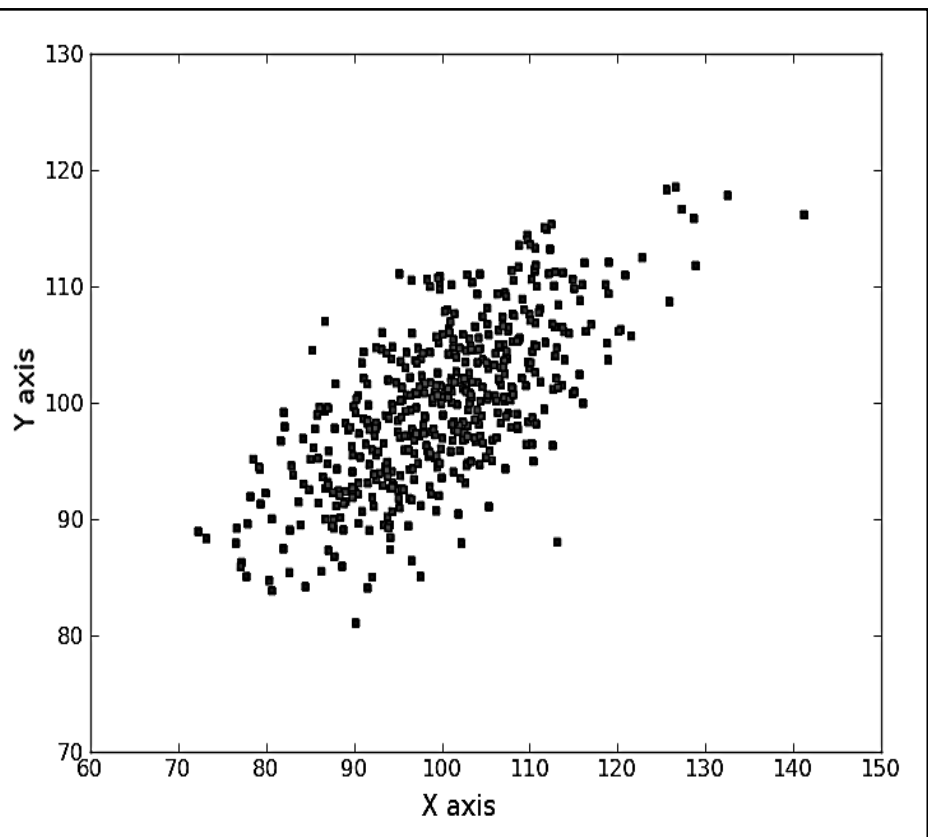

Figure 4. The overall noise associated with the PSD system in the $X$ and $Y$ axis of the PSD.

It can be noticed from Fig. 4 that the noise is not Gaussian in effect and have diagonal effects to it.

These simulations suggest that the noise implications of the system are strongly degraded based on the amplification system as previously known [12].

Choosing a PSD that does not adhere to the tetra-lateral effect such as a lateral effect PSD is an ideal substitution because of the resolution of approximately $0.5 \mu \mathrm{m}$. This was suggested by some of the researchers in the area [13-15]. This is shown in the post-processing of the PSD signal [16] and has applications in the robotics localization [17].

The initial result of the localization is shown in Fig. 6. It is clear that without the proposed method, the signal is not square shaped.

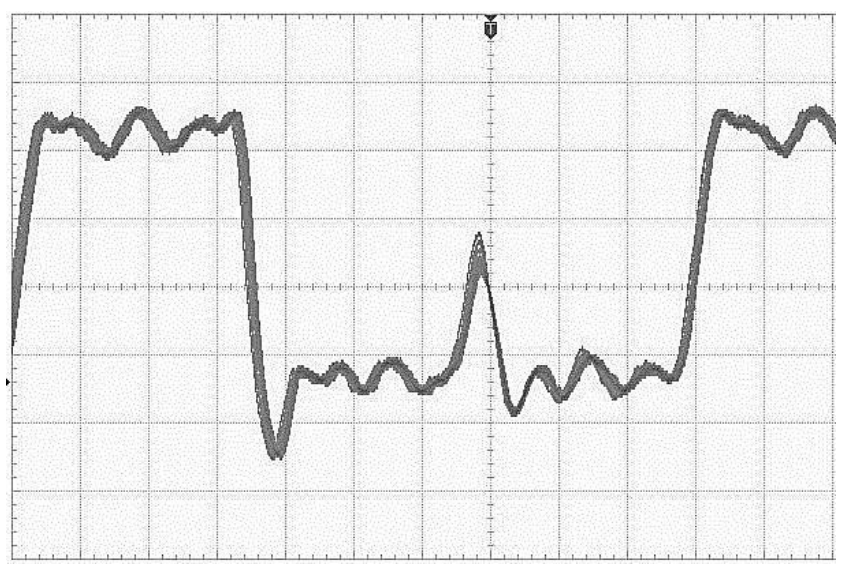

Figure 6. Without the localization proposed method, the signal is not square shaped and shows a significant amount of noise. The figure is from the Oscilloscope connected to the output of the circuit.

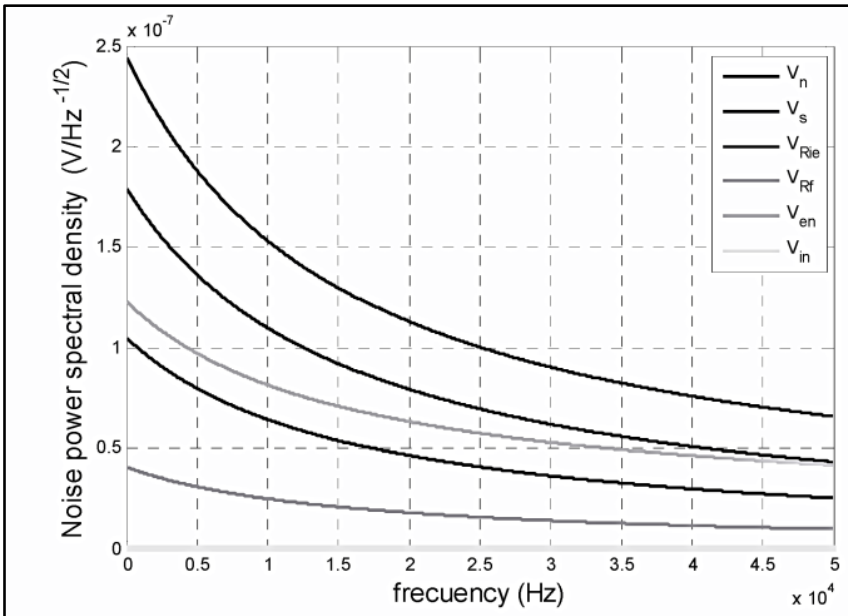

(a)

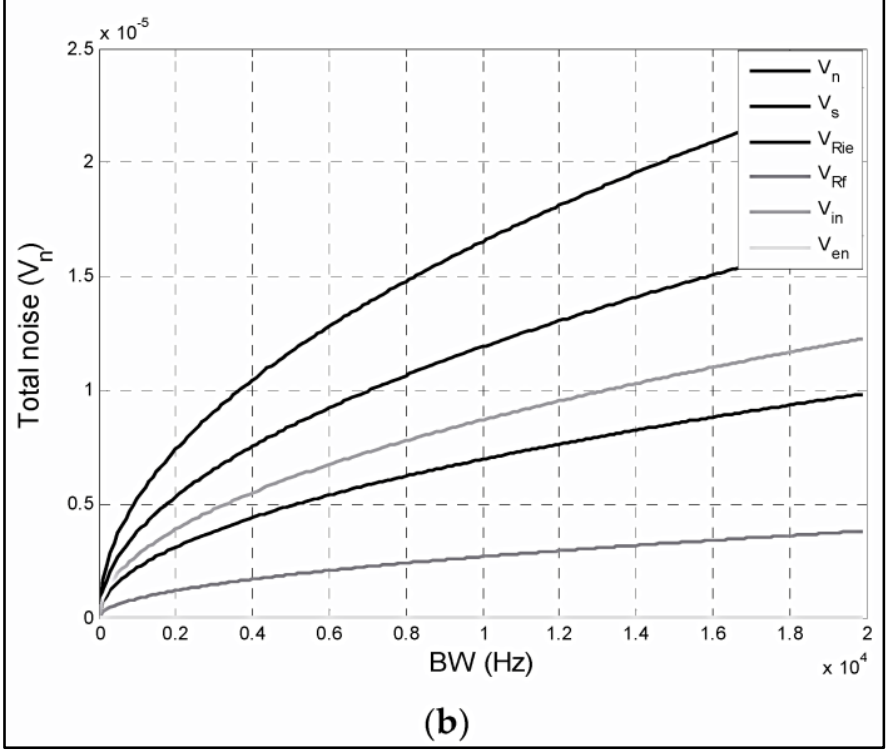

Figure 5. The noise power in contrast to the frequency and the bandwidth $(B W)$. 


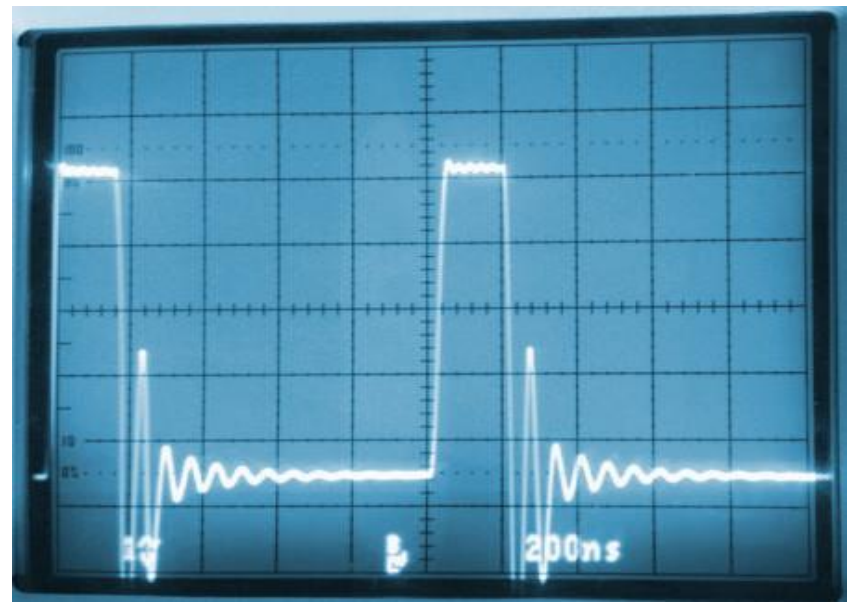

Figure 7. This figure shows the 2nd improvement on the signal based on the proposed method. The square shape verifies the proposed method.

This result was improved significantly by employing a localized differential method as is shown in Fig. 7.

It is clear from Fig. 7 that the proposed method is reducing the associated noise to a large degree. This is very consistent with the result of the other research in the area [18].

To further improve this result, the differential localization can be employed to rectify the small noises remained in the system. Fig. 8 shows that final implementation result.

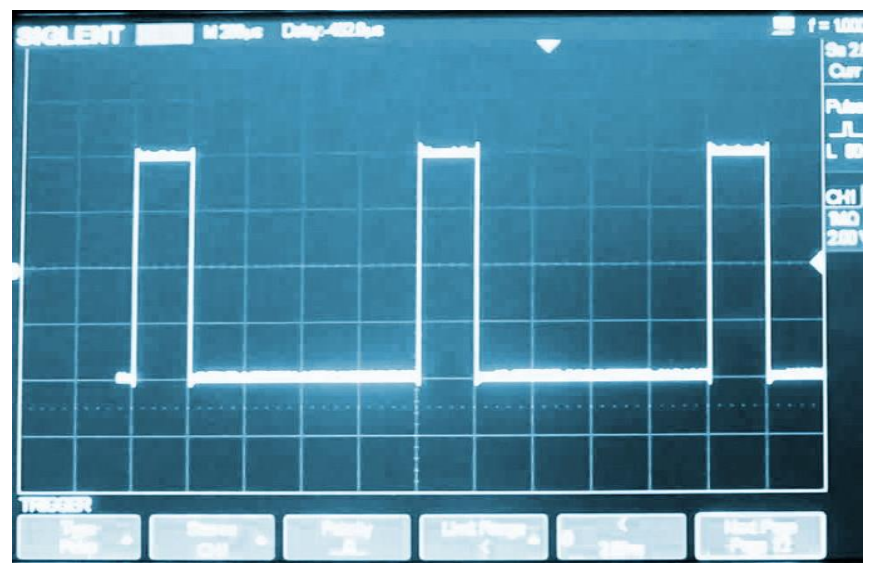

Figure 8. The final improvement of the noise associated with the system is shown and the rectification is significantly improved using the differential localization method

The final result presented in Fig. 8 shows significant improvement in the noise reduction of the system in excess of $140 \%$ in comparison to our previous tests where the filtering was not applied (Fig. 6). This has shown to improve the precision of the PSD system to a very large degree.

It is shown in the simulation and the actual implementation of the system that the noise can be reduced significantly to improve the precision of the system.

\section{CONCLUSION}

This research presents our proposed method of localized differential approach to improving the precision of the PSD system. It was shown through the simulation and the actual implementation of the system that the noise associated with overall system was significantly reduced.

The post-processing of the lateral PSD signal was shown to be the most effective approach on improving the signal to noise ratio.

Another improvement was the implementation of the Villiers approach to the Brown's model of rectification of the pin-cushion type distortion.

Overall, the simulation and actual result show a very significant improvement in the precision of the PSD system.

\section{FURTHER STUDIES}

Because of the photocurrent nature of the PSD, the leaking of the current to the electrode was seen as a noticeable issue in our implementation. This may be prevented by employing a digitalized system by using an Analog to Digital Converter (ADC) in relation to the amplifier system.

Some future research that can be considered by the use of the localized differential approach conducted in this paper include implementing a $2^{\text {nd }}$ degree filter based on the differential method and also investigating the effects of this filter on duo-lateral PSDs as so as tetra-lateral PSDs. The same can be also investigated in a one-dimensional PSD. A comparison between these implementations can help with future research in this field.

\section{ACKNOWLEDGEMENT}

This research was supported by the Singapore Research Program (SRP2018-9741-F2RE). Authors thank the research program from the National University of Singapore.

\section{AUTHOR CONTRIBUTION}

Venky Dijkkomp and Kanai Ogale designed the system and conducted the simulation and participated in the implementation with equal shared as much as the contribution to writing the paper.

\section{DECLARATION}

Authors have disclosed no conflicts of interests. 


\section{REFERENCE}

[1] Salido-Monzú, D.; Martín-Gorostiza, E.; LázaroGalilea, J.L.; Domingo-Pérez, F.; Wieser, A. Multipath mitigation for a phase-based infrared ranging system applied to indoor positioning. In Proceedings of the 2013 International Conference on Indoor Positioning and Indoor Navigation, Montbeliard-Belfort, France, 28-31 October 2013; pp. 1-10.

[2] Jung, S.-Y.; Hann, S.; Park, C.-S. TDOA-based optical wireless indoor localization using LED ceiling lamps. IEEE Trans. Consum. Electron. 2011, 57, 1592-1597.

[3] Rahimi, Mehdi, and Yantao Shen. "PSD microscopy: a new technique for adaptive local scanning of microscale objects." Robotics and biomimetics 4 , no. 1 (2017): 6.

[4] Rahimi, Mehdi, and Yantao Shen. "Accelerated adaptive local scanning of complicated micro objects for the PSD scanning microscopy: methods and implementation." In International conference on intelligent autonomous systems, pp. 511-521. Springer, Cham, 2016.

[5] Rodríguez-Navarro, David, José Luis Lázaro-Galilea, Álvaro De-La-Llana-Calvo, Ignacio Bravo-Muñoz, Alfredo Gardel-Vicente, Georgios Tsirigotis, and Juan Iglesias-Miguel. "Indoor Positioning System Based on a PSD Detector, Precise Positioning of Agents in Motion Using AoA Techniques." Sensors 17, no. 9 (2017): 2124.

[6] M. Rahimi, and Y. Shen. "Flash scanning: An ultra fast local scanning of complicated objects for PSD microscopy using 2D bisection." In Real-time Computing and Robotics (RCAR), 2017 IEEE International Conference on, pp. 721-726. IEEE, 2017.

[7] Cui, Song, and Yeng Chai Soh. "Linearity indices and linearity improvement of 2-D tetralateral position-sensitive detector." IEEE Transactions on Electron Devices 57, no. 9 (2010): 2310-2316.A 2-D tetra-lateral PSDone-dimensional PSD consisting of a uniform P-type resistive layer,

[8] Rahimi, M., Luo, Y., Harris, F.C., Dascalu, S.M. and Shen, Y., 2014, December. Improving measurement accuracy of position sensitive detector (PSD) for a new scanning PSD microscopy system. In Robotics and Biomimetics (ROBIO), 2014 IEEE International Conference on (pp. 1685-1690). IEEE.

[9] Brown, D.C., 1966. Decentering Distortion of Lenses. Photometric Engineering, 32(3), pp.444-462.

[10] De Villiers JP, Leuschner FW, Geldenhuys R. Centipixel accurate real-time inverse distortion correction. InOptomechatronic Technologies 20082008 Nov 17
(Vol. 7266, p. 726611). International Society for Optics and Photonics.

[11] Seyed Mehdi Rahimi. "Towards Developing a Scanning Position Sensitive Detector (PSD) Microscopy: PSD Measurement Enhancement, Adaptive Local Scanning and Implementation." PhD diss., 2016.

[12] Wait, J.V., Huelsman, L.P. and Korn, G.A., 1975. Introduction to operational amplifier theory and applications (pp. 293-294). New York: McGraw-Hill.

[13] Hongfei, Liu, Xiao Ying, and Chen Zhong. "A high precision optical position detector based on duolateral PSD." In Computer Science-Technology and Applications, 2009. IFCSTA'09. International Forum on, vol. 3, pp. 90-92. IEEE, 2009.

[14] Blank, S., Shen, Y., Xi, N., Zhang, C. and Wejinya, U.C., 2007, October. High precision PSD guided robot localization: Design, mapping, and position control. In Intelligent Robots and Systems, 2007. IROS 2007. IEEE/RSJ International Conference on (pp. 52-57). IEEE.

[15] M. Rahimi and Y. Shen, 2014, September. Adaptive local scanning: a comprehensive and intelligent method for fast scanning of indiscrete objects. In Multisensor Fusion and Information Integration for Intelligent Systems (MFI), 2014 International Conference on (pp. 1-6). IEEE.

[16] Wang, Q., Xia, J., Liu, X. and Zhao, Y., 2015. Novel Method of Detecting Movement of the Interference Fringes Using One-Dimensional PSD. Sensors, 15(6), pp.12857-12871.

[17] Park, Jae-Han, Dae-Hee Won, Ki-Young Park, Seung-Ho Baeg, and Moon-Hong Baeg. "Development of a real time locating system using PSD under indoor environments." In SICE-ICASE, 2006. International Joint Conference, pp. 4251-4255. IEEE, 2006.

[18] Rodríguez-Navarro, David, José Luis Lázaro-Galilea, Ignacio Bravo-Muñoz, Alfredo Gardel-Vicente, Francisco Domingo-Perez, and Georgios Tsirigotis. "Mathematical Model and Calibration Procedure of a PSD Sensor Used in Local Positioning Systems." Sensors 16, no. 9 (2016): 1484. 